\title{
Growth and Characterization of Ultrathin Fe Films on Molecule-Adsorbed MgO Surfaces
}

\author{
Takayuki Kojima*1, Masaki Mizuguchi, Kohei Oka, Seiji Mitani*2 and Koki Takanashi \\ Institute for Materials Research (IMR), Tohoku University, Sendai 980-8577, Japan
}

Effects of the molecule-adsorption on a $\mathrm{MgO}$ surface on the growth of ultra-thin Fe films were investigated. Surface observation by reflection high-energy electron diffraction and ultra-high vacuum atomic force microscopy showed that the adsorption of molecules on the MgO surface affected the morphology of Fe films grown on the $\mathrm{MgO}$ surface. It was revealed that the surface flatness of Fe films was improved by appropriate molecule-adsorption on the $\mathrm{MgO}$ surface. [doi:10.2320/matertrans.M2009218]

(Received June 25, 2009; Accepted August 17, 2009; Published October 7, 2009)

Keywords: ultra-thin film, molecule-adsorption, epitaxial growth, atomic force microscopy

\section{Introduction}

Ultra-thin films have attracted a lot of attention from the viewpoint of understanding physics in two-dimensional electron systems. Two-dimensional electron systems are formed typically in semiconductor hetero-junctions. These systems show interesting phenomena, such as Shubnikov-de Haas oscillation ${ }^{1)}$ and quantum Hall effect ${ }^{2)}$ at very low temperatures. Two-dimensional electron systems formed in ultra-thin metallic films also show quantum effects due to interference and confinement of electrons. It is known that thin films of ferromagnetic metals clearly exhibit spindependent quantum well states. ${ }^{3,4)}$ In previous studies, ultrathin metallic films were grown on metallic buffer layers. If the ultra-thin metallic films are grown on an insulator, the further development of spintronic devices such as spinpolarized resonant tunneling transistors which are demonstrated by gate-control of spin-dependent quantum well states is expected. ${ }^{5,6)}$ However, the growth of extremely thin metallic films with a few atomic layers on insulators is quite difficult because three-dimensional island structures are easily formed. Hence, only a few studies on the growth of ultra-thin metallic films on insulators have been reported to date. $^{7-11)}$

In the first stage of film growth, the metals that have higher surface energies than that of an insulating substrate are generally grown three-dimensionally. The migration of metallic atoms promotes the formation of island structure, and not continuous structure. The growth of metallic thin films using adsorption of atoms or molecules on surfaces of insulators was reported. ${ }^{12,13)} \mathrm{M}$. Lust et al. reported that crystalline qualities of ultrathin $\mathrm{Fe}$ and $\mathrm{Cu}$ films grown on $\mathrm{MgO}$ were improved by adsorption of $\mathrm{CO}$ molecules on a $\mathrm{MgO}$ surface. ${ }^{14)}$ In addition, it was reported that dissociative adsorption of $\mathrm{H}_{2} \mathrm{O}$ molecules occurs on defect sites of the $\mathrm{MgO}$ surface, which seems to be an advantage to grow epitaxial films on $\mathrm{MgO}$ substrates. ${ }^{15,16)}$ Therefore, the

\footnotetext{
${ }^{* 1}$ Graduate Student, Tohoku University

*2Present address: National Institute for Materials Science (NIMS), Tsukuba 305-0047, Japan
}

adsorption of $\mathrm{CO}$ or $\mathrm{H}_{2} \mathrm{O}$ molecules on the $\mathrm{MgO}$ surface has a possibility of realizing the growth of ultra-thin continuous ferromagnetic films.

In this paper, we have investigated the effects of molecule-adsorption on a $\mathrm{MgO}$ surface on the growth of ultra-thin Fe films. $\mathrm{CO}$ and $\mathrm{H}_{2} \mathrm{O}$ were adsorbed on $\mathrm{MgO}$ surfaces, and Fe films with a nominal thickness of a few atomic layers were grown on the molecule-adsorbed surfaces. Surface morphology was observed by reflection highenergy electron diffraction and atomic force microscopy. A characteristic feature in this study is that these direct methods were used to investigate effects of adsorption.

\section{Experimental Procedure}

All the growth processes were performed at room temperature by molecular beam epitaxy with evaporation sources in an ultra-high vacuum (UHV) chamber (base pressure: $\left.1 \times 10^{-7} \mathrm{~Pa}\right)$. $\mathrm{MgO}(001)$ substrates were annealed at $600^{\circ} \mathrm{C}$ for $30 \mathrm{~min}$ in $\mathrm{UHV}$, and $\mathrm{MgO}$ buffer layers $(5 \mathrm{~nm})$ were grown at room temperature. Then, clean $\mathrm{MgO}(001)$ surfaces were obtained by annealing at $600^{\circ} \mathrm{C}$ for $30 \mathrm{~min}$. Ultra-thin Fe films with a nominal thickness of $0.3 \mathrm{~nm}$ were grown on the $\mathrm{MgO}$ surfaces. The $\mathrm{CO}$-adsorbed $\mathrm{MgO}$ surface was prepared by introduction of $\mathrm{CO}$ gas with a partial pressure of $4 \times 10^{-6} \mathrm{~Pa}$ from a variable leak valve for 10 min equipped in the UHV chamber. The growth of Fe film was started after closing the valve, and the partial pressure of $\mathrm{CO}$ gas decreased to the order of $10^{-8} \mathrm{~Pa}$. To adsorb $\mathrm{H}_{2} \mathrm{O}$ molecules on a $\mathrm{MgO}$ surface, the $\mathrm{MgO}$ substrate with the buffer layer mentioned above was unloaded from the UHV chamber, and dipped into ultra-pure water for $5 \mathrm{~s}$. The $\mathrm{H}_{2} \mathrm{O}$-adsorbed $\mathrm{MgO}$ surface was annealed again at $100^{\circ} \mathrm{C}$ for $3 \mathrm{~min}$ in the UHV chamber, and an Fe film was grown on the surface. The surface morphology was monitored by reflection high-energy electron diffraction (RHEED) just after deposition and after annealing. The surface profile and the surface flatness of the Fe films were investigated by ultra-high vacuum atomic force microscopy (AFM) using a contact mode. 

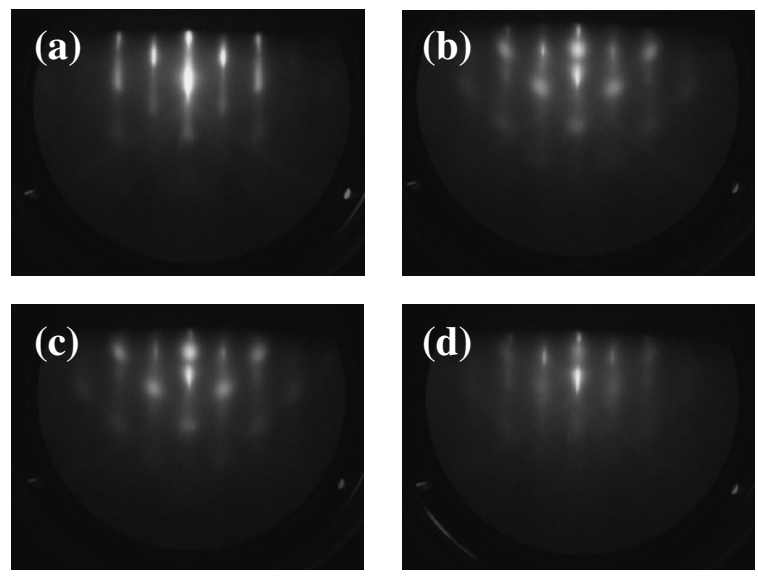

Fig. 1 RHEED patterns for (a) a $\mathrm{MgO}(001)$ buffer layer after annealing, and for Fe layers with a thickness of $0.3 \mathrm{~nm}$ just after deposition on (b) non-adsorbed $\mathrm{MgO}$, (c) $\mathrm{CO}$-adsorbed $\mathrm{MgO}$, (d) $\mathrm{H}_{2} \mathrm{O}$-adsorbed $\mathrm{MgO}$. The incident beam was directed along the azimuth of $\mathrm{MgO}\langle 110\rangle$.

\section{Results and Discussion}

Figure 1(a) shows a RHEED pattern from a MgO buffer layer after annealing. A streaky pattern, which indicates the formation of a flat surface, was observed. An Fe film grown on the non-adsorbed $\mathrm{MgO}$ surface shows a spotty pattern as shown in Fig. 1(b). This RHEED pattern indicates the epitaxial island growth of bcc-Fe with an epitaxial orientation relationship of $\mathrm{Fe}\langle 110\rangle / / \mathrm{MgO}\langle 100\rangle$. A similar spotty pattern was also observed for the $\mathrm{Fe}$ layer grown on the CO-adsorbed $\mathrm{MgO}$ surface (Fig. 1(c)). A RHEED pattern from the Fe layer grown on the $\mathrm{H}_{2} \mathrm{O}$-adsorbed $\mathrm{MgO}$ surface revealed a slightly fuzzy pattern as shown in Fig. 1(d). This implies a poor crystalline quality of the Fe film.

Figure 2 shows AFM images of the surfaces of a $\mathrm{MgO}$ buffer layer and Fe films. In Fig. 2(a) which shows the surface of the $\mathrm{MgO}$ buffer layer, where bright particle-like regions and dark voids dispersed over the entire surface are observed. On the surface of an Fe film grown on nonadsorbed $\mathrm{MgO}$ as shown in Fig. 2(b), a similar image to Fig. 2(a) is observed. On the surface of an Fe film grown on $\mathrm{CO}$-adsorbed $\mathrm{MgO}$ as shown in Fig. 2(c), a number of distinct voids are observed. On the surface of an Fe film grown on $\mathrm{H}_{2} \mathrm{O}$-adsorbed $\mathrm{MgO}$ as shown in Fig. 2(d), the numbers of bright regions and voids have been reduced. No apparent difference in morphology is seen in this view-scale between the $\mathrm{MgO}$ buffer layer (Fig. 2(a)) and the Fe film grown on non-adsorbed $\mathrm{MgO}$ (Fig. 2(b)). The number of bright regions in Fig. 2(c) seems to be smaller than that in Fig. 2(b). The bright regions are not observed in Fig. 2(d). These results reveal that the adsorption of molecules such as $\mathrm{CO}$ or $\mathrm{H}_{2} \mathrm{O}$ on the $\mathrm{MgO}$ surface apparently affects the morphology of the $\mathrm{Fe}$ thin film. Magnified images of Figs. 2(a), (b), (c) and (d) are shown in Figs. 2(e), (f), (g) and (h), respectively. Figure 2(f) indicates that small $\mathrm{Fe}$ particles were grown on non-adsorbed $\mathrm{MgO}$. Fe particles are also observed in the magnified images of the Fe films grown on CO-adsorbed $\mathrm{MgO}$ (Fig. 2(g)) and on $\mathrm{H}_{2} \mathrm{O}$-adsorbed $\mathrm{MgO}$ (Fig. 2(h)). It is found that bright regions in Fig. 2(b), (c) and (d) consist of a number of Fe particles. The sizes of $\mathrm{Fe}$
Table 1 Surface roughness $(R a)$ of a $\mathrm{MgO}$ buffer layer and Fe films grown on various $\mathrm{MgO}$ surfaces.

\begin{tabular}{ccccc}
\hline \multirow{2}{*}{$R a / \mathrm{nm}$} & $\mathrm{MgO}$ & \multicolumn{3}{c}{ Ultra-thin Fe films } \\
\cline { 3 - 5 } & buffer & Non-ad. & CO-ad. & $\mathrm{H}_{2} \mathrm{O}$-ad. \\
\hline $\begin{array}{c}\text { All area } \\
\begin{array}{c}\text { Excl. } \\
\text { voids region }\end{array}\end{array}$ & 0.79 & 1.0 & 0.89 & 0.72 \\
\hline
\end{tabular}

particles seem to be almost the same for all the Fe films. The existence of Fe particles shows that even Fe thin films grown molecular-adsorbed $\mathrm{MgO}$ surfaces form three-dimensional islands.

Surface roughness $(R a)$ of the $\mathrm{MgO}$ buffer layer and the $\mathrm{Fe}$ films grown on respective $\mathrm{MgO}$ surfaces is summarized in Table 1. Here, $R a$ is defined as an arithmetic average of the absolute values of heights. $R a$ is expressed as

$$
R a=\frac{1}{x_{\max } y_{\max }} \int_{0}^{x_{\max }} \int_{0}^{y_{\max }}\left|z(x, y)-z_{0}\right| d x d y,
$$

where $x_{\max }$ and $y_{\max }$ are side lengths of an arbitrary rectangle region, and $z(x, y)$ and $z_{0}$ are the height at $(x, y)$ and the average height in the whole region, respectively. $R a$ of the Fe film grown on non-adsorbed $\mathrm{MgO}$ is larger than that of the $\mathrm{MgO}$ buffer layer. On the other hand, $R a$ 's of Fe films grown on $\mathrm{CO}$-adsorbed and $\mathrm{H}_{2} \mathrm{O}$-adsorbed $\mathrm{MgO}$ are smaller than that on non-adsorbed $\mathrm{MgO}$. Particularly, $R a$ 's of both the Fe films estimated by excluding void regions are considerably smaller than those of the $\mathrm{MgO}$ buffer layer and of the $\mathrm{Fe}$ film on non-adsorbed $\mathrm{MgO}$. This indicates that the surface flatness is improved by molecule-adsorption on the $\mathrm{MgO}$ surface.

The results shown above reveal the improvement of the surface flatness of Fe films by the adsorption of $\mathrm{CO}$ and $\mathrm{H}_{2} \mathrm{O}$ on $\mathrm{MgO}$. A possible mechanism for this effect is considered as follows. Fe islands are three-dimensionally grown on $\mathrm{MgO}$, because the surface energy of Fe is higher than that of $\mathrm{MgO}$. Therefore, the migration of $\mathrm{Fe}$ atoms promotes the aggregation of $\mathrm{Fe}$ particles. In the case of $\mathrm{CO}$-adsorption and $\mathrm{H}_{2} \mathrm{O}$-adsorption, the migration of $\mathrm{Fe}$ atoms is possibly blocked by $\mathrm{CO}$ or $\mathrm{H}_{2} \mathrm{O}$ molecules. As a result, the formation of rough islands is suppressed, and the surface flatness is improved. In order to elucidate more details on the mechanism, however, all the experiments should be performed in-situ, and precisely controllable introduction of $\mathrm{H}_{2} \mathrm{O}$ molecules is needed.

\section{Summary}

The effect of molecule-adsorption on the $\mathrm{MgO}$ surface on the growth of ultra-thin Fe films have been investigated. It has been revealed that the adsorption of $\mathrm{CO}$ and $\mathrm{H}_{2} \mathrm{O}$ on the $\mathrm{MgO}$ surface apparently influences the morphology of $\mathrm{Fe}$ thin films. Although small Fe particles are grown, the surface flatness of Fe films is improved by the adsorption of $\mathrm{CO}$ or $\mathrm{H}_{2} \mathrm{O}$. This result indicates a possibility of the growth of an ultra-thin continuous Fe film with an atomically flat surface by optimizing a suitable condition of the moleculeadsorption. 

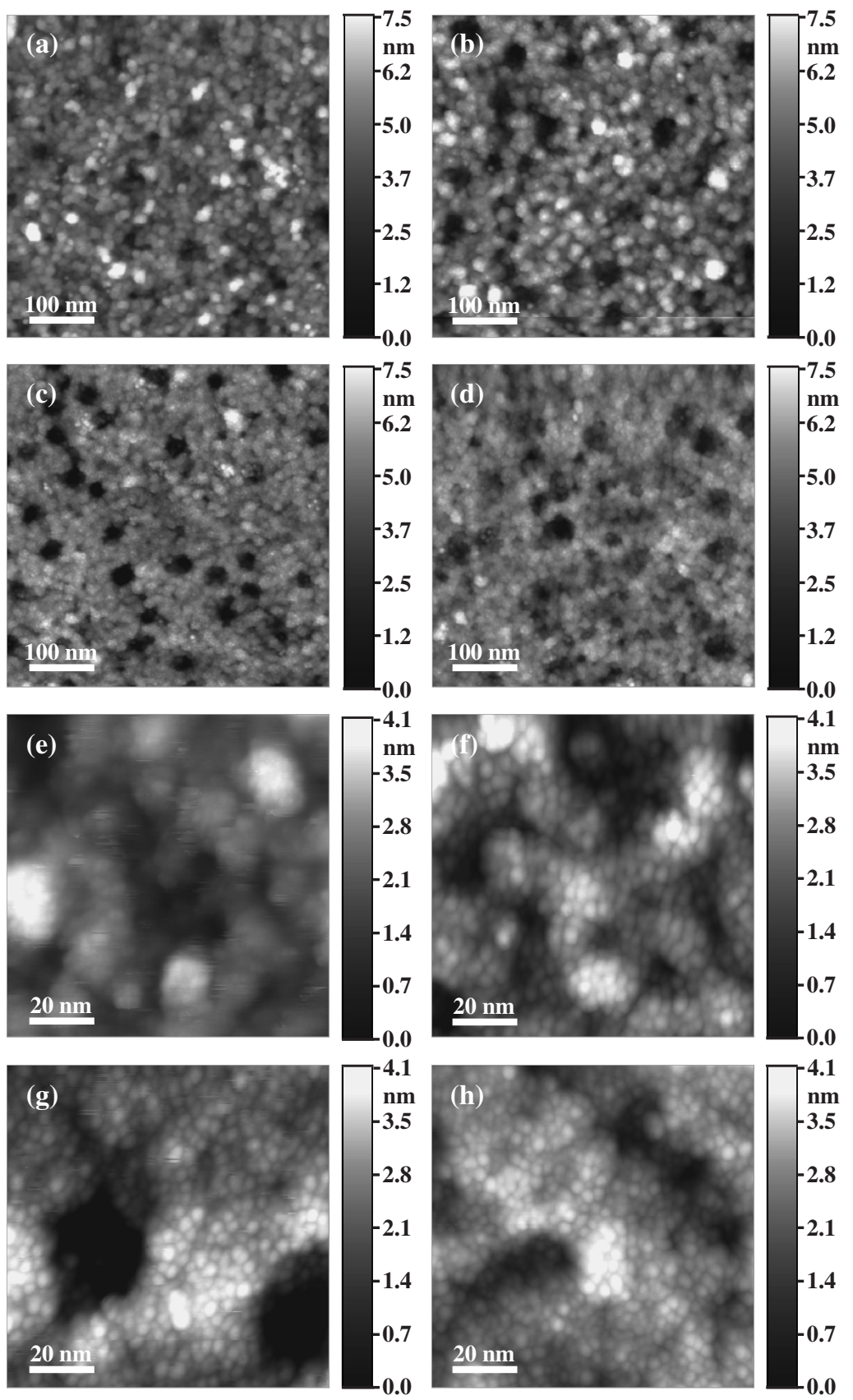

Fig. 2 AFM images of the surface of (a) a MgO buffer layer and Fe layers with a thickness of 0.3 nm grown on (b) non-adsorbed MgO,

(c) CO-adsorbed $\mathrm{MgO}$, (d) $\mathrm{H}_{2} \mathrm{O}$-adsorbed $\mathrm{MgO}$. (e), (f), (g) and (h) are magnified images of (a), (b), (c) and (d), respectively.

\section{REFERENCES}

1) L. L. Chang, H. Sakaki, C. A. Chang and L. Esaki: Phys. Rev. Lett. 38 (1977) 1489-1493.

2) K. v. Klitzing, G. Dorda and M. Pepper: Phys. Rev. Lett. 45 (1980) 494-497.

3) Y. Suzuki, T. Katayama, S. Yoshida, K. Tanaka and K. Sato: Phys. Rev. Lett. 68 (1992) 3355-3358.

4) T. Katayama, Y. Suzuki and W. Geerts: J. Magn. Magn. Mater. 5364 (1996) 158-162.

5) X. Zhang, B.-Z. Li, G. Sun and F.-C. Pu: Phys. Rev. B 56 (1997) 5484-5488.

6) T. Nozaki, N. Tezuka and K. Inomata: Phys. Rev. Lett. 96 (2006) 027208.

7) M. Jałochowski, M. Hoffman and E. Bauer: Phys. Rev. Lett. 76 (1996) 4227-4229.
8) M. Henzler, T. Lüer and A. Burdach: Phys. Rev. B 58 (1998) 1004610053.

9) O. Pfennigstorf, K. Lang, H.-L. Günter and M. Henzler: Appl. Surf. Sci. 162-163 (2000) 537-546.

10) V. Zielasek, H. Liu, A. A. Shklyaev, E. P. Rugeramigabo and H. Pfnür: Phys. Rev. B 72 (2005) 115422.

11) V. Hnatyuk and M. Stróżak: J. Phys.: Condens. Matter 19 (2007) 396001.

12) H.-P. Steinrück, F. Pesty, L. Zhang and T. E. Madey: Phys. Rev. B 51 (2007) 2427-2439.

13) T. Lewowski and P. Wieczorek: Appl. Surf. Sci. 93 (1996) 85-87.

14) M. Lust, A. Priebe, G. Fahsold and A. Pucci: Surf. Interface Anal. 33 (2002) 487-490.

15) C. A. Scamehorn, N. M. Harrison and M. I. McCarthy: J. Chem. Phys. 101 (1994) 1547-1554.

16) M. J. Stirniman, C. Huang, R. S. Smith, S. A. Joyce and B. D. Kay: J. Chem. Phys. 105 (1996) 1295-1298. 